\title{
A general stress patterning method for manufacturing ultra-precise free- form deformation in thin-substrates (Conference Presentation) (Withdrawal Notice)
}

Youwei Yao, Brandon Chalifoux, Ralf Heilmann, Mark Schattenburg

Youwei Yao, Brandon Chalifoux, Ralf Heilmann, Mark Schattenburg, "A general stress patterning method for manufacturing ultra-precise free-form deformation in thin-substrates (Conference Presentation) (Withdrawal Notice)," Proc. SPIE 11822, Optics for EUV, X-Ray, and Gamma-Ray Astronomy X, 118220W (16 August 2021); doi: 10.1117/12.2595537

Event: SPIE Optical Engineering + Applications, 2021, San Diego, California, United States 


\section{A general stress patterning method for manufacturing ultra- precise free-form deformation in thin-substrates (Withdrawal Notice)}

Youwei Yao, Brandon Chalifoux, Ralf Heilmann, Mark Schattenburg

Proceedings Volume 1 1822, Optics for EUV, X-Ray, and Gamma-Ray Astronomy X; $118220 \mathrm{~W}$ (2021)

https://doi.org/10.1117/12.2595537

Event: Optics for EUV, X-Ray, and Gamma-Ray Astronomy X, 2021, San Diego, California, United States

Online Publication Date: 5 August 2021

Withdrawn from Publication: 13 August 2021

Publisher's Note: This conference presentation, originally published on 5 August 2021 was withdrawn on 13 August 2021 per author request. 